\title{
Proposta de um aplicativo de Realidade Aumentada para tratamento de aracnofobia
}

\author{
Karoline Dias Barreto ${ }^{1}$, Fabiano Fagundes ${ }^{1,2}$, Cristina D'Ornellas Filipakis Souza ${ }^{1,2}$, \\ Lauriane dos Santos Moreira ${ }^{2}$ \\ ${ }^{1}$ Ciência da Computação - ${ }^{2}$ Psicologia - Centro Universitário Luterano de Palmas \\ (CEULP/ULBRA) Avenida Teotônio Segurado, CEP 77.019-900 - Palmas - TO - Brasil \\ \{karol.db1230, thilfa\}@gmail.com, \{cristina, lauriane\}@ceulp.edu.br
}

\begin{abstract}
Resumo. Tendo em vista que as fobias são um medo aprendido, do ponto de vista da análise comportamental, e que uma fobia pode ser ocasionada devido a estímulos aversivos, é necessário pensar em formas de confrontar o medo através da emissão de respostas assertivas. A exposição in vivo é um tratamento que costuma ser recomendado para Fobias Específicas - como a aracnofobia. Entretanto, este apresenta alta taxa de conflito, ou seja, os indivíduos podem vir a achar que esse tipo de exposição é muito assustador. Nesse sentido, a Realidade Aumentada surge como um meio alternativo para ser utilizado. O presente trabalho utilizou recursos ofertados pela Realidade Aumentada juntamente com conceitos de análise do comportamento, tendo como foco a dessensibilização sistemática.
\end{abstract}

\section{Introdução}

O medo é um comportamento emocional que, quando prejudicial para o indivíduo e para o grupo ao qual ele está inserido, é denominado pela literatura psiquiátrica como sendo fobia (FORNAZARI; BORLOTI; HAYDU, 2014). Um dos transtornos de ansiedade, segundo o DSM - IV-TR (Manual de Diagnóstico e Estatística de Perturbações Mentais), no qual a pessoa desenvolve um medo descomedido, persistente ou irracional e sempre relacionado a uma situação ou objeto específico é chamado de Fobia Específica. (LOPES; CARVALHO; BARBOSA, 2014). A aracnofobia, medo de aranhas, é uma das Fobias Específicas.

Segundo Lima et al. (2013), reabilitações que utilizam a exposição in vivo (exposição do fóbico frente ao seu medo) podem desencadear ataques de pânico, resultando no não prosseguimento do tratamento. Com base nisso, há a necessidade de outras formas de exposição. A exposição a uma Fobia Específica sendo mediada por uma tecnologia (neste caso a Realidade Aumentada) pode ajudar na percepção pelo fóbico de forma mais racional.

A dessensibilização sistemática surge a partir da análise do comportamento como uma técnica de ajuda que consiste em repetir situações consideradas ameaçadoras de maneira gradual, de forma que, com o avanço do tratamento, o estado de desconforto no qual a pessoa se encontra possa diminuir.

Diante do exposto, entende-se que é necessário buscar meios alternativos de tratamento. Por misturarem o mundo real com o virtual, sistemas de Realidade Aumentada são apontados como forma de tratamento para confrontar a sensação fóbica. O fóbico pode, dessa forma, estar em contato com a sua fobia tendo conhecimento que o que está sendo exposto não é real.

\section{Fobias}

O medo é uma emoção comum sentida pelas pessoas. A emoção é definida por Skinner (2000, p. 182) em sua obra "Ciência e Comportamento Humano" como um estado particular de alta ou baixa frequência, decorrente de uma ou mais respostas que são induzidas por qualquer uma XXI Encoinfo - Congresso de Computação e Tecnologias da Informação ISSN: 2447-0767 
entre uma classe de operações (conjuntos de meios que se combinam para obter-se um resultado).

Segundo Vilela (2005 apud VARGAS, OLIVEIRA e RIBEIRO, 2008), fobia (de phobos, deusa grega do medo) pode ser estabelecida como um medo insistente e irracional, resultando no ato de esquivar-se de uma situação, atividade e/ou objetos específicos temidos.

Com o objetivo de aliviar os sintomas que as fobias provocam, há tratamentos com a utilização de medicamentos ou, até mesmo, com a utilização de tecnologias. A terapia comportamental, por outro lado, remete às terapias de exposição e conclui que uma exposição prolongada e controlada (sem consequências físicas) ao que causa a fobia, pode, dessa forma, diminuir de maneira progressiva os sintomas fóbicos (RAMOS; RIOS; SERRANO, 2018).

Para os behavioristas, o indivíduo é considerado uma folha em branco e, a partir de ações externas, passa por um processo de aprendizagem e experimentação que o condiciona a comportamentos. Portanto, as fobias, seriam, de certa forma, um comportamento aprendido decorrente de influências externas.

Fobia Específica tem como característica medos irracionais que são associados a estímulos específicos, desencadeando uma forte reação de ansiedade. Um batracofóbico, que tem medo da família de anfíbios, incluindo sapos e rãs, pode vir a enxergá-los maiores, mais feios e mais repugnantes do que eles de fato são.

Devido a Fobia Específica interferir menos nas funções pessoais ou estar relacionada a um sofrimento mais leve, os fóbicos não buscam atendimento médico psiquiátrico. $\mathrm{O}$ tratamento pode ser procurado pelos indivíduos em períodos que situações da vida podem tornar a fobia problemática, por exemplo. (LIPSITZ et al., 1999).

A pessoa que possui uma fobia, neste caso uma Fobia Específica, tende a ter um pensamento deturpado por considerar que algumas situações são mais ameaçadoras do que realmente aparentam ser. (ARAUJO, 2011).

Os aracnofóbicos, foco deste trabalho, apresentam medo intenso e imediato quando expostos a aranhas. Estas pessoas conseguem identificar que o medo é excessivo e/ou irracional, mas tal percepção não as tornam aptas a contê-lo. Portanto, passam a evitar situações que os exponham a aranhas.

O medo de aranhas pode vir a afetar a vida pessoal e profissional dos indivíduos aracnofóbicos, visto que pessoas com aracnofobia podem ter sua rotina afetada e, conforme afirmam Granado, Ranvaud e Peláez (2007), em casos mais extremos, o medo de ser confrontado pelo objeto fóbico chega a ser tão grande que as pessoas se negam a passar por qualquer terapia.

Existem técnicas que procuram confrontar a sensação fóbica, como a exposição ao vivo e a dessensibilização sistemática. Segundo Turner (1996, apud REY, 2002), a exposição ao vivo é uma técnica comportamental que tem como intuito expor o fóbico direta e gradualmente a objetos e situações que causam temor. Geralmente nessa técnica não se utilizam técnicas de relaxamento.

\section{Análise do Comportamento}

A análise do comportamento tem seu surgimento a partir do behaviorismo radical e seus princípios. Behaviorismo (ou comportamentalismo) tem como principal objeto de estudo o comportamento.

Para entender a análise do comportamento é necessário compreender o comportamento. Para explicar o comportamento, Skinner (1974) levanta questionamentos separando-os em duas questões práticas: 1- como uma pessoa poderia se antecipar e a partir disso estar preparada para a ação de outra pessoa? e 2- como poderia-se induzir uma pessoa a uma outra forma de se comportar? Dessa maneira, o comportamento poderia ser entendido como uma questão sobre causas.

\footnotetext{
112 XXI Encoinfo - Congresso de Computação e Tecnologias da Informação ISSN: 2447-0767
} 
Em sua obra "Questões Recentes na Análise Comportamental”, Skinner (2002) afirma que confundir sentimentos com causa é fácil, pois nós os sentimos enquanto estamos nos comportando (ou anteriormente ao comportamento em si). O sentimento, para Skinner, é um tipo de ação sensorial, porque podemos ver alguma coisa e senti-la também. Porquanto, a maneira como os indivíduos se sentem é constantemente tão importante quanto o que eles fazem (SKINNER, 2002, p.13-22).

Para Prette e Prette (2017), a análise do comportamento tem uma abordagem filosófica, científica e aplicada que excede o controle dos comportamentos sociais, não podendo ser reduzida a habilidades sociais (diferentes abordagens teóricas com foco em comportamentos sociais).

A partir do entendimento sobre comportamento e análise do comportamento surge a Terapia Comportamental. Segundo Nogueira (2013), esta tem como objetivo a análise de relações entre ambiente e comportamento. A Terapia Comportamental utiliza uma técnica que trabalha diferentes comportamentos, chamada de dessensibilização sistemática.

\section{Dessensibilização sistemática}

A dessensibilização sistemática, conforme Knapp e Caminha (2003), é composta por um conjunto de técnicas de exposição ao que ocasionou o trauma. A técnica de dessensibilização tem como objetivo expor uma pessoa a um estímulo que este descrevia como sendo causador de sua ansiedade juntamente com um estado de relaxamento. Esta técnica, portanto, poderia vetar a reação emocional frente ao estímulo. (BARBOSA; BORBA, 2010).

Diante disso, Turner (2002, ARAUJO, 2011), afirma que através de emissão de respostas assertivas, a dessensibilização procurar eliminar comportamentos de medo e evitação.

Segundo Brochado (2002), a dessensibilização sistemática possui três passos básicos:

- Passo 1: o indivíduo deve formular uma lista de estímulos que provocam medo (juntamente com o terapeuta). Esses estímulos, posteriormente, serão classificados hierarquicamente (hierarquia do medo), de maneira que situações que provam menos medo se posicionam no ponto mais baixo e as que provocam mais medo são inseridas em posições mais elevadas.

- Passo 2: neste passo o indivíduo deve aprender e treinar maneiras de relaxar, utilizando técnicas de relaxamento. Quando as situações de hierarquia de medo forem apresentadas, tendo conseguido dominar a técnica de relaxamento, esta deve conseguir bloquear o medo.

Passo 3: O indivíduo é apresentado, gradualmente, às situações de medo. Neste passo, o terapeuta realiza a descrição verbal das situações com a ajuda de ilustrações ou materiais que sejam reais. Cada situação é, portanto, trabalhada até que a pessoa não tenha a sensação de medo.

\section{Realidade Aumentada}

A Realidade Aumentada possibilita que o usuário visualize o mundo real de maneira aumentada por meio de elementos virtuais. A exposição in vivo costuma ser o tratamento recomendado para Fobias Específicas, mas, por apresentar alta taxa de conflito (alguns indivíduos podem achar esse tipo de exposição muito assustador), não é eficaz em todas as instâncias. Nesse sentido, a Realidade Aumentada se mostra como uma forma de exposição mais aceitável para alguns indivíduos (BOTELLA et al., 2010). 
Com a utilização da Realidade Aumentada, é possível visualizar elementos que são virtuais (como a projeção de animais 3D) como se estivessem ao alcance das mãos, por meio de um aplicativo no celular, por exemplo. A exposição a uma Fobia Específica através desta tecnologia pode ajudar na percepção, pelo fóbico, de uma forma mais racional, visto que o indivíduo tem a percepção de que está lidando com objetos não reais em um mundo real.

Um exemplo de aplicação de Realidade Aumentada que ficou conhecida em todo o mundo é o jogo Pokémon Go, produzido pela Nintendo. O jogo, por sua vez, combina a geolocalização com a Realidade Aumentada, unindo os mundos real e virtual. Para o seu funcionamento, é necessário acionar a câmera que posiciona de forma virtual, em um determinado local, criaturas (Pokémon) que efetivamente não estão lá (AGRELA, 2016). A Figura 1 apresenta a utilização deste jogo em um smartphone.

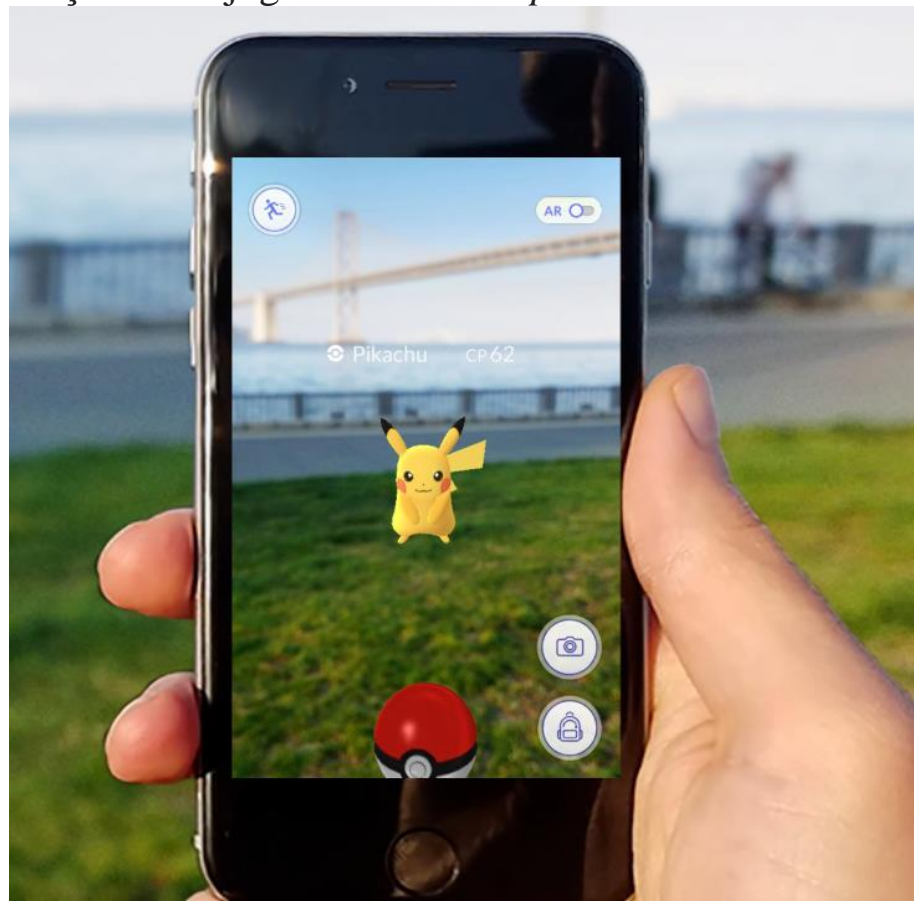

Figura 1: Utilização do Pokémon Go em um smartphone

Fonte: Visualização do app Pokemon Go (POKEMONGO, 2019)

Com a utilização da Realidade Aumentada, o profissional psicólogo terá o controle sobre os elementos que serão necessários para viabilizar a dessensibilização sistemática. É necessário salientar que a exposição com a utilização da Realidade Aumentada, assim como as exposições imaginárias e in vivo, necessita ser mediada por um psicólogo, que terá a responsabilidade de monitorar o indivíduo que está sendo exposto ao tratamento e verificar suas reações ansiosas frente a exposição.

\section{Materiais e Métodos}

O GamingPlace é o aplicativo móvel onde os jogos construídos poderão ser jogados. Já que os jogos são feitos de acordo com as necessidades de cada usuário, apenas os usuários que forem explicitamente autorizados pelo criador de cada jogo devem ter acesso a este, portanto, para que o sistema possa mostrar apenas os jogos corretos. ao acessar o aplicativo pela primeira vez, o jogador deverá informar seu nome de usuário e senha, dessa forma o GamingPlace enviará à API uma requisição para fazer login e, caso esta obtenha sucesso, suas credenciais de acesso serão salvas em banco de dados local (para que não seja necessário que o usuário faça login todas as vezes que desejar utilizar o aplicativo), serão mostradas ao usuário suas informações de perfil e serão listados os jogos disponíveis a ele. 
Para o desenvolvimento do presente trabalho estão sendo utilizadas as seguintes tecnologias e materiais:

- Unity: uma plataforma em tempo real (realiza a renderização em tempo real) que possui ferramentas e serviços e é utilizada para criação de jogos e aplicativos;

- Vuforia: um kit de desenvolvimento de software (SDK) que possibilita a integração da Realidade Aumentada a aplicativos para desktop e dispositivos móveis, oferecendo suporte a diferentes plataformas e sistemas operacionais, como o Android e IOS. Esta utiliza o rastreamento e reconhecimento de marcadores ou imagens pré configuradas que possibilitam o posicionamento de um objeto $3 \mathrm{D}$ em tempo real (BERGAMASCHI; MORAIS, 2014);

- Linguagem de programação $\mathrm{C \#}$ : linguagem que será utilizada para a codificação do projeto;

- Visual Studio Code: editor de código que será utilizado para a programação dos scripts (conjuntos de instruções) que serão introduzidos no projeto da Unity.

A composição do processo de desenvolvimento do trabalho é apresentada na Figura 2 a seguir.

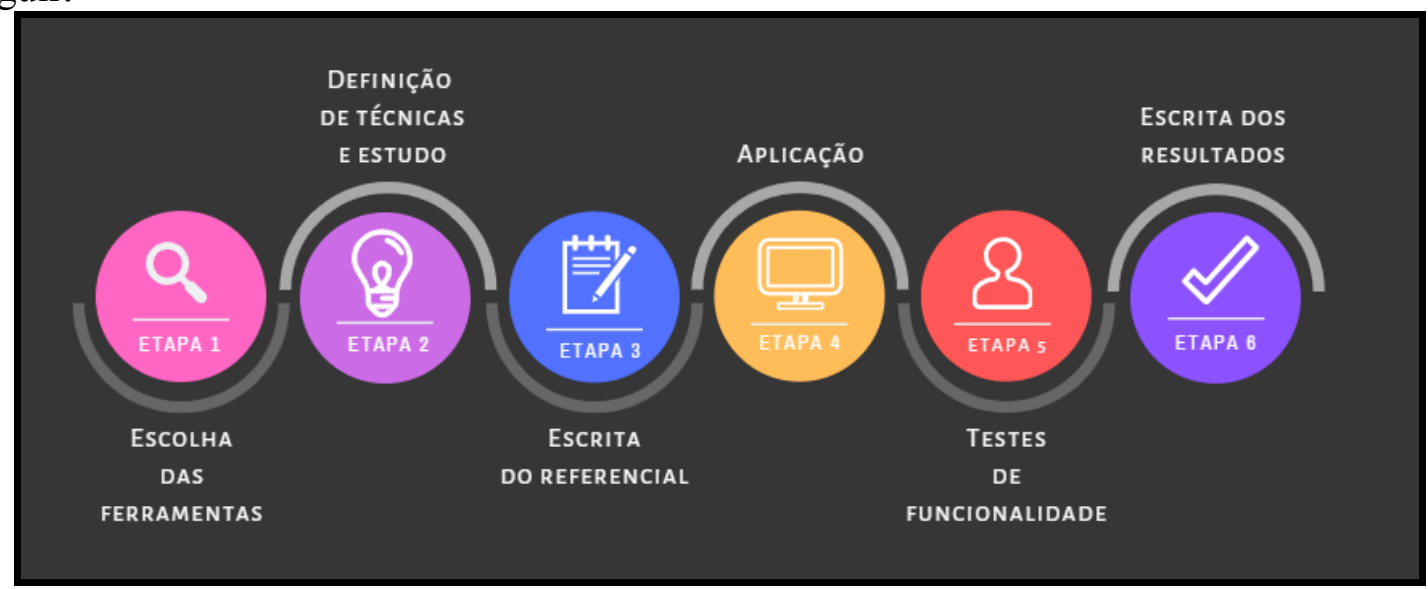

Figura 2: Processo de desenvolvimento do trabalho

A Figura 2 apresenta o processo de desenvolvimento do trabalho. A primeira etapa é constituída da escolha das ferramentas que serão utilizadas para a criação do aplicativo de Realidade Aumentada: Unity, Vuforia, linguagem de programação C\# e Visual Studio Code. $\mathrm{Na}$ segunda etapa foi realizada a definição de técnicas e estudo das mesmas, unindo conceitos da área da psicologia como análise comportamental com foco na dessensibilização sistemática.

A etapa seguinte corresponde à escrita do referencial, abordando os conceitos: Fobias, Fobias Específicas, Aracnofobia, Análise do Comportamento, Dessensibilização Sistemática e Realidade Aumentada.

A quarta etapa é de aplicação, ou seja, desenvolvimento da aplicação, estando esta dividida em:

- modelagem do aplicativo: estruturação da utilização da dessensibilização sistemática juntamente com a Realidade Aumentada

- montagem: organização da sequência de etapas que conterão no aplicativo.

- programação das cenas que irão compor o sistema

Como quinta etapa o aplicativo contará com testes de funcionalidade e, como última etapa, os resultados obtidos serão descritos a partir do desenvolvimento da aplicação e dos testes de funcionalidade que serão aplicados. Testes da aplicabilidade do aplicativo 
considerando o aspecto psicológico serão realizados em trabalhos futuros desenvolvidos no curso de Psicologia do CEULP.

\section{Resultados}

Para o desenvolvimento de um aplicativo no qual é desejável criar várias cenas e nessas cenas colocar objetos (modelos) diferentes, o projeto na Unity é criado e a partir dele as diversas cenas com seus respectivos modelos são criados. A Unity possibilita, também, a criação de botões, que podem ser utilizados para interação com o usuário e textos, que aparecem na tela e também podem ser utilizados para comunicação com o usuário que está utilizando o aplicativo.

Assim, a Figura 3 ilustra um exemplo de uma aplicação 3D desenvolvida com o Unity. Na cena há a inserção de um modelo 3D de uma aranha.

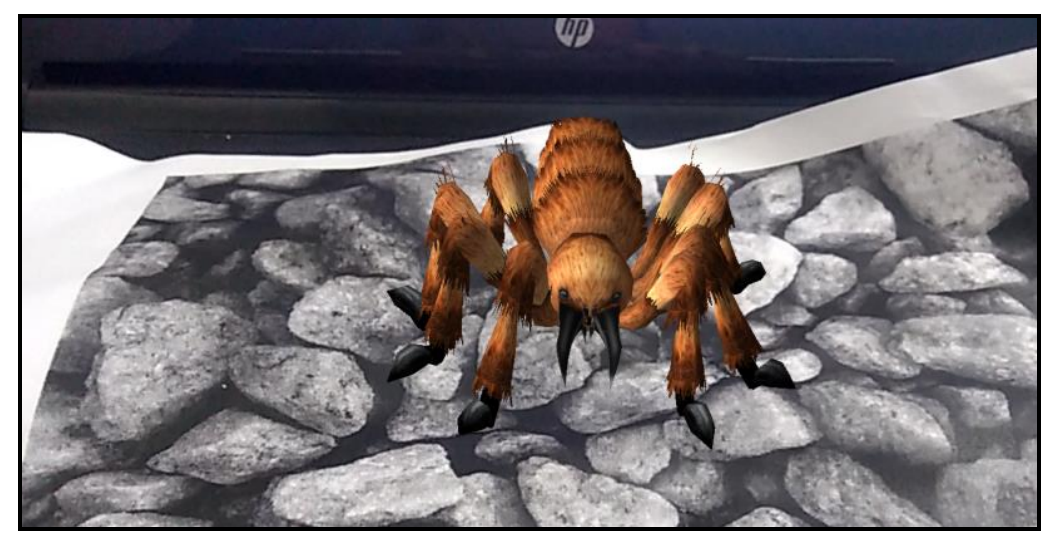

Figura 3: Aranha 3D projetada pelo aplicativo desenvolvido na Unity

A aranha que está sendo projetada é renderizada em cima de uma imagem (foto de pedras) utilizando conceitos e características da Realidade Aumentada. Para que isso possa acontecer, a Vuforia é integrada ao projeto da Unity, possibilitando a criação de aplicações de Realidade Aumentada. Para o desenvolvimento de uma aplicação de Realidade Aumentada é necessário, ao primeiro passo, criar uma ARCamera (Figura 4), que é instalada através do Vuforia Engine. Este componente, que é a câmera de Realidade Aumentada, permite que, a partir de uma base de dados, as imagens 3D inseridas na cena sejam projetadas. 


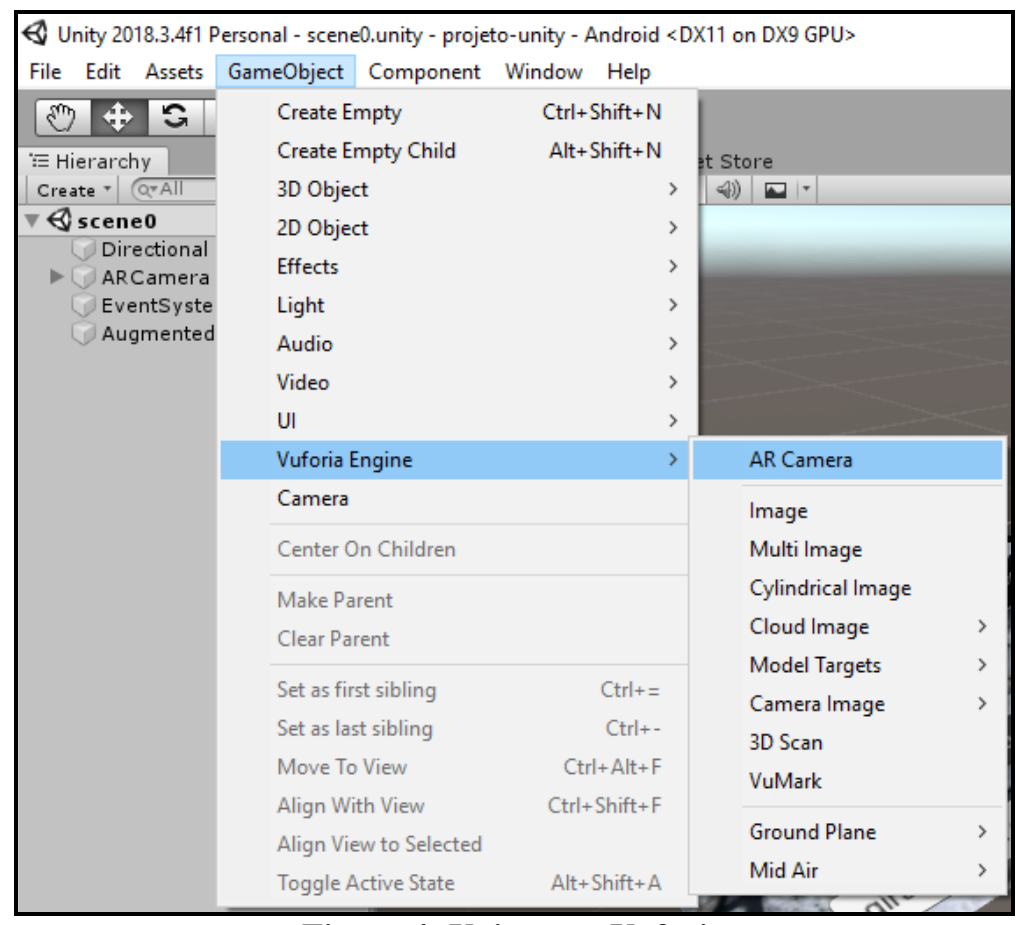

Figura 4: Unity com Vuforia

Portanto, para que fosse possível visualizar a aranha na Figura 3 apresentada anteriormente, há uma base de dados cadastrada na Vuforia com uma imagem de pedras. Essa base de dados é exportada para o Unity para ser utilizada na cena, como a Figura 5 ilustra.

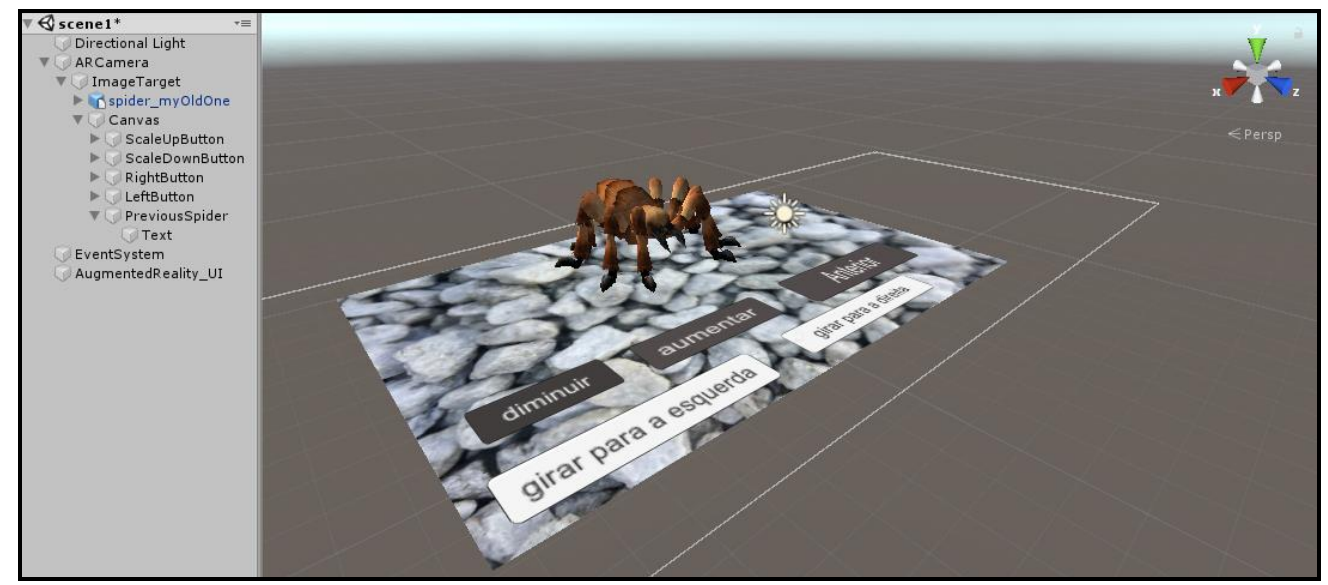

Figura 5: Exemplo de uma cena no Unity

A imagem de pedras é adicionada na cena através do ImageTarget (elemento da cena) e dentro desta são inseridos o modelo 3D da aranha e os botões diminuir, aumentar, anterior, girar para a esquerda e girar para a direita. Para que estes elementos sejam apresentados, uma imagem igual a que foi cadastrada a base de dados deve ser imprimida e apresentada frente à câmera, que pode ser uma webcam ou a câmera do celular, por exemplo.

O aplicativo está sendo projetado com a utilização da técnica de dessensibilização sistemática, sendo composto, dessa forma, por etapas. Como a dessensibilização sistemática aborda uma exposição gradual ao que ocasionou a fobia, o aplicativo irá conter etapas que vão desde apresentar o nome "aranha" até apresentar uma aranha de maneira estática e em outro momento uma aranha em movimento, ou interagindo com o usuário.

É necessário salientar que este aplicativo só poderá ser aplicado e utilizado com a presença de um profissional da psicologia, que irá monitorar o usuário de acordo com seus níveis de ansiedade. Isso facilita ao profissional psicólogo pois ele contará, facilmente, com 
os elementos necessários para promover a dessensibilização sistemática e, em especial, terá controle sobre estes elementos.

\section{Considerações Finais}

O presente trabalho teve como propósito apresentar uma proposta de um aplicativo para tratamento de aracnofobia, com a utilização dos recursos da Realidade Aumentada em conjunto com conceitos da análise comportamental, tendo como foco a dessensibilização sistemática.

Os tratamentos ofertados como a exposição in vivo podem não surtir efeito em algumas situações, visto que o fóbico pode apresentar ataques de pânico e não prosseguir com o tratamento, o que acarreta na necessidade de outras formas de exposição. A tecnologia da Realidade Aumentada é apresentada, portanto, como uma maneira de exposição, combinando os mundos real e virtual.

Durante o desenvolvimento do trabalho, as etapas relacionadas ao processo foram seguidas para a construção de um aplicativo que englobasse os conceitos da psicologia, com a utilização da técnica dessensibilização sistemática. É importante ressaltar que a aplicação do mesmo necessita da presença de um profissional da psicologia, para que o usuário que está sendo exposto à tecnologia possa ser monitorado.

O presente trabalho que está em andamento terá como próximas etapas: a modelagem do aplicativo, a organização da sequência de etapas que conterão no aplicativo e a implementação do aplicativo. Por fim, testes de funcionalidade serão aplicados ao aplicativo com o objetivo de verificar se este está de acordo com o esperado, em relação as suas funcionalidades.

\section{Referências}

AGRELA, Lucas. O que é realidade aumentada, chave do sucesso de Pokémon Go. 2016. Disponível em: <https://exame.abril.com.br/tecnologia/o-que-e-realidade -aumentada-chave-do-sucesso-de-pokemon-go/>. Acesso em: 14 abr. 2019.

ARAUJO, Neuraci Gonçalves de. Fobia específica: passo a passo de uma intervenção bemsucedida. Revista Brasileira de Terapia Comportamental e Cognitiva, Rio de Janeiro, v. 7, n. 2, p. 37-45, dez. 2011. Disponível em: $<$ http://pepsic.bvsalud.org/scielo.php?script=sci_arttext\&pid=S1808 $-56872011000200007 \& \operatorname{lng}=$ pt\&nrm=iso $>$. acessos em 18 mar. 2019.

BARBOSA, João Ilo Coelho; BORBA, Aécio. O surgimento das terapias cognitivocomportamentais e suas consequências para o desenvolvimento de uma abordagem clínica analítico-comportamental dos eventos privados. Revista Brasileira de Terapia Comportamental e Cognitiva, [s.1.], v. 12, n. 1/2, p.60-79, 16 jun. 2010. Revista Brasileira de Terapia Comportamental e Cognitiva (RBTCC). http://dx.doi.org/10.31505/rbtcc.v12i1/2.416. Disponível em: $<$ http://www.usp.br/rbtcc/index.php/RBTCC/article/view/416/310 >. Acesso em: 30 mar. 2019.

BERGAMASCHI, Dr. Marcelo Pereira; MORAIS, Thamires Martins Augusto de. Estudo sobre a utilização de VUFORIA e Unity 3D com RA para dispositivos móveis. WRVA 2014 Workshop de Realidade Virtual e Aumentada, Marília - Sp, nov. 2014. Disponível em: <http://www.lbd.dcc.ufmg.br/colecoes/wrva/2014/031.pdf >. Acesso em: 1 abr. 2019.

BOTELLA, Cristina et al. Treating Cockroach Phobia With Augmented Reality. Behavior Therapy, [s.1.], v. 41, n. 3, p.401-413, set. 2010. Elsevier BV. http://dx.doi.org/10.1016/j.beth.2009.07.002.

BROCHADO, Monica Maria Viviani. O Medo no Esporte. Motriz, v.8, n.2, p.69-77, 2002. 
FORNAZARI, Verônica Bender Haydu Silvia Aparecida; BORLOTI, Elizeu; HAYDU, Nícholas Bender. Facetas da Exposição In Vivo e por Realidade Virtual na Intervenção Psicológica no Medo de Dirigir. Psico, Porto Alegre, v. 2, n. 45, p.1-11, abr./jun. 2014.

GRANADO, Laura Carmilo; RANVAUD, Ronald; PELÁEZ, Javier Ropero. A Spiderless Arachnophobia Therapy: Comparison between Placebo and Treatment Groups and SixMonth Follow-Up Study. Neural Plasticity, [s.1.], v. 2007, p.1-11, 2007. Hindawi Limited. http://dx.doi.org/10.1155/2007/10241.

KNAPP, Paulo; CAMINHA, Renato Maiato. Terapia cognitiva do transtorno de estresse póstraumático. Revista Brasileira de Psiquiatria, [s.1.], v. 25, n. 1, p.31-36, jun. 2003. FapUNIFESP (SciELO). http://dx.doi.org/10.1590/s1516-44462003000500008.

LIMA, Lazaro V. de O. et al. Sistema para auxiliar o tratamento de Aracnofobia usando Realidade Aumentada- usabilidade centrada no terapeuta. Computer On The Beach, Florianópolis - $\quad$ Sc, p.268-277, 2013. Disponível em: $<$ https://siaiap32.univali.br/seer/index.php/acotb/article/viewFile/6223/3484>. Acesso em: 10 abr. 2019.

LOPES, C.c.; CARVALHO, S.c.; BARBOSA, M.r.. Tratamento de fobia específica por dessensibilização e reprocessamento por meio dos movimentos oculares. Psicologia: Teoria e Prática, [s.1.], v. 16, n. 1, p.31-42, 11 abr. 2014. GN1 Genesis Network. http://dx.doi.org/10.15348/1980-6906/psicologia.v16n1p31-42.

NOGUEIRA, Samira Brito. Superação do Medo de Janela por Meio da Dessensiblização Sistemática. Psicologado. ed. 04/2013. Disponível em < https://psicologado.com.br/abordagens/comportamental/superacao-do-medo-de-janela-pormeio-da-dessensiblizacao-sistematica >. Acesso em 27 Mar 2019.

POKEMONGO. https://pokemongolive.com/pt_br/. 2019. Acesso em 27 Mar 2019.

PRETTE, Zilda Aparecida Pereira del; PRETTE, Almir del. Habilidades sociais e análise do comportamento. Perspectivas em Análise do Comportamento, [s.1.], v. 1, n. 2, p.104-115, 23 ago. 2017. Associação Paradigma - Centro de Ciências e Tecnologia do Comportamento. http://dx.doi.org/10.18761/perspectivas.v1i2.33.

RAMOS, Olga; RÍOS, Daniel; SERRANO, Jan. Treatment of Specific Phobia by using Exposure Therapy through Virtual Reality. International Journal Of Applied Engineering Research, India, v. 13, n. 15, p.12018-12022, 2018. Disponível em: $<$ https://www.ripublication.com/ijaer18/ijaerv13n15_44.pdf $>$. Acesso em: 28 mar. 2018.

REY, Gustavo J. Fonseca D'el et al. Exposição ao vivo no tratamento de agorafobia: relato de caso. Psicologia: Ciência e Profissão, [s.1.], v. 22, n. 4, p.80-85, dez. 2002. FapUNIFESP (SciELO). http://dx.doi.org/10.1590/s1414-98932002000400010.

SKINNER, B. F. (1974). About behaviorism. New York, NY: Alfred A. Knopf.

SKINNER, B.F. Questões Recentes na Análise Comportamental. 3 ed. Campinas: Papirus, 2002.

SKINNER, B.F. Ciência e Comportamento Humano. São Paulo: Martins Fontes, 2000.

LIPSITZ, Joshua D. et al. Specific phobia 10-16 years after treatment. Depression And Anxiety, [s.1.], v. 10, n. 3, p.105-111, 1999. Wiley. http://dx.doi.org/10.1002/(sici)15206394(1999)10:33.0.co;2-x.

VARGAS, Geovana C.; OLIVEIRA, Isabel Cristina V. de; RIBEIRO, Karla Carolina S.. Freud e Hitchcock: comparação de quadros de fobia. Latin American Journal of Fundamental Psychopathology On Line, São Paulo, v. 5, n. 1, p. 56-68, mai. 2008. Disponível em: $<$ http://pepsic.bvsalud.org/scielo.php?script=sci_arttext\& pid=S1677-03582008000100006\&lng=pt\&nrm=iso $>$. Acesso em: 18 mar. 2019. 